\title{
Enhanced user performance in an image gallery application with a mobile autostereoscopic touch display
}

\author{
Anni Sassi ${ }^{a}$, Perttu Pöyhönen ${ }^{a}$, Sini Jakonen ${ }^{a}$, Sini Suomi ${ }^{a}$, Tolga Capin ${ }^{b}$, Jukka Häkkinen ${ }^{\text {a,* }}$ \\ a Institute of Behavioral Sciences, P.O. Box 9, FI00014 University of Helsinki, Finland \\ ${ }^{\mathrm{b}}$ Department of Computer Engineering, Bilkent University, Turkey
}

\section{A R T I C L E I N F O}

\section{Article history:}

Received 21 October 2013

Received in revised form 2 May 2014

Accepted 5 May 2014

Available online 14 May 2014

\section{Keywords:}

Interaction

Mobile device

Autostereoscopic display

Touch display

Image gallery

Visual search

\begin{abstract}
A B S T R A C T
In this study, we explored how stereoscopic depth affects performance and user experience in a mobile device with an autostereoscopic touch display. Participants conducted a visual search task with an image gallery application on three layouts with different depth ranges. The task completion times were recorded, and the participants were asked to rate their experiences. The results revealed that the image search times were facilitated by a mild depth effect and that too great a depth slowed search times and decreased user-experience ratings.
\end{abstract}

(c) 2014 Elsevier B.V. All rights reserved.

\section{Introduction}

Today, the popularity of 3D media on mobile devices is increasing, and devices with three-dimensional autostereoscopic displays are becoming more common. The possibilities of autostereoscopic displays that do not require additional glasses for the stereoscopic effect are intriguing, especially for mobile devices. Stereoscopic three-dimensionality offers the possibility of utilizing the small display area of a mobile device in an efficient manner in 3D user interfaces and applications such as 3D digital content consumption and 3D maps. The limitations of the mobile context, including the small physical screen size and limited input modalities, can be overcome to some degree by interactions in 3D.

There are several studies supporting the idea that an autostereoscopic display brings added value to the user interface of a mobile device $[1,2]$. Stereo effects on an autostereoscopic display have been reported to improve the viewing experience and naturalness of the visual content [3]. One study found that in a visual search task, stereoscopic depth can reduce the impact of the visual crowding effect [4].

\footnotetext{
* Corresponding author. Tel.: +358 504839483.

E-mail addresses: anni.sassi@iki.fi (A. Sassi), perttu.poyhonen@gmail.com (P. Pöyhönen), sini.jakonen@helsinki.fi (S. Jakonen), sinimaarit.suomi@gmail.com (S. Suomi), tcapin@cs.bilkent.edu.tr (T. Capin), jukka.hakkinen@helsinki.fi (J. Häkkinen).
}

Interactivity is a key feature for 3D user experience on mobile devices. It is desirable that the user engage with $3 \mathrm{D}$ content actively instead of being a passive consumer. Several user-input alternatives are currently available on mobile devices, including touchscreen-based input, inertial trackers, and camera-based tracking. Each has its advantages and disadvantages. Among them, touchscreen-based interfaces have emerged as the standard input technique for mobile devices. Because touch-based interaction provides a direct means of interacting with 3D content by directly touching and manipulating 3D graphical elements, it is also a natural and appealing style of input for 3D applications.

However, there are several difficulties related to combining touch-based input with autostereoscopic mobile displays. In stereoscopic displays, each eye is expected to see a slightly different perspective of the scene. If the user is to interact with the 3D user interface, the question arises as to where they would touch the $2 \mathrm{D}$ surface to select the 3D elements. Furthermore, for single- or multi-touch screen UIs, the main limitation is that interactive elements should be presented in at least a $1 \times 1 \mathrm{~cm}$ square on the touch surface to be selected by an average finger. In return, this limits how many UI elements can be rendered on the display.

In this paper, we investigate the usability of a three-dimensional image gallery application on a mobile device equipped with lenticular autostereoscopic display. The user experience is compared with a layout in which three rows have different depths, which creates an amphitheater-type layout. The visual search task 
completion times are reported, and participants' subjective imagequality and user-experience evaluations are reported.

\section{Background}

\subsection{Cognitive issues of stereoscopic depth on $3 D$ displays}

A stereoscopic display increases the efficiency with which a small display can be utilized, as stereoscopic 3D (S3D) presentation offers better visualization possibilities. Stereoscopy, for example, reduces pattern recognition ambiguity in occluded, layered, and transparent visualizations [5] and, thus, decreases visual clutter that might be present in a situation where there is overlapping information in the display. For example, in map applications, S3D presentation would be a very useful feature, as text and symbols would be easier to discriminate from the geographical information [6]. In addition to decluttering the display, objects with large enough stereoscopic depth differences are processed more efficiently $[7,8]$ and are less affected by visual crowding [4,9], which might be a problem with a small display. There may also be other cognitive benefits in using stereoscopic 3D, as it has been suggested that a 3D interface might increase performance because the spatial localization of objects would make memorization and recall easier [10-12].

Although stereo can be beneficial in user interfaces, it should be utilized with care, as there are restrictions within human stereoscopic vision that must be taken into account so that viewing the user interface is a fun and comfortable experience. Depth magnitudes and depth gradients are especially important, as depth values that are too high or depth gradients that are too steep are difficult to watch and cause eye strain [13-15]. Furthermore, large disparities might slow down the processing due to the increased time required for stereoscopic fusion and convergence movements. Conversely, depth values that are too small contribute no added value for the user $[1,16]$, so values that are not too large or too small should be utilized. In the current experiment, the disparity values were so small that we did not expect that the processing would be slowed by these factors.

An issue specifically related to stereoscopic touch displays is that the discrepancy between the physical surface and virtual 3D UI elements might be problematic. When a 3D UI element has a distant depth, the user must press the physical surface in front of the element. When the 3D UI object has a near depth, the user must press the physical surface through the button. The greater the depth of the button, the more this discrepancy affects the interaction [17]. In the current experiment, we used small disparities, so the effect of this discrepancy was expected to be small.

\subsection{Autostereoscopic mobile displays}

The technical implementation of a stereoscopic user interface and the chosen technology can significantly affect the viewing experience. Separate viewing glasses are not viable for a mobile device that should be easy to carry, so an autostereoscopic display that works without glasses is the most promising option for these devices. Autostereoscopic displays can be implemented as parallax barriers or lenticular displays (Fig. 1), where the main feature of such a display is that it exhibits an interleaved image so that one image is shown to left eye and another image to right eye. The lenticular sheets are composed of small lenses with particular shapes that refract the light in specific directions. The shapes are cylindrical or spherical to enable the proper light redirection. A parallax barrier is essentially a mask with openings and solid regions that block the light in specific directions. Autostereoscopic displays halve the horizontal resolution of the image, which is an important restriction with a small mobile display.
Both technologies have certain limitations. The viewer should be placed within a restricted area, called a sweet spot, to see the stereoscopic effect. Moving outside this proper area, the user might observe the opposite views and experience so-called pseudoscopy. Non-ideal separation between views creates inter-view cross-talk, manifested in ghost-like images. Furthermore, the crosstalk can increase as a function of disparity [18], which was visible in our lenticular display. We conducted expert testing before the actual experiments and selected small disparity values, as larger disparities would have impaired the recognition of the image and potentially caused difficulties in stereoscopic depth perception [19].

\section{3. $3 D$ User interfaces}

According to the application and the targeted task, different 3D UI layout techniques can be selected. Undeniably, depth positioning adds complexity to the design of UIs because more layout options emerge. A stereoscopic 3D UI looks quite different from a 3D UI rendered on a 2D screen. To designers without significant prior experience with the characteristics of stereoscopic design, guessing the visual effects of positioning UI elements in depth can be difficult.

Three-dimensional user interfaces have been studied previously, but these studies have dealt with non-stereoscopic threedimensionality. Some of the investigated interfaces have, in fact, been $2 \frac{1}{2} \mathrm{D}$, allowing the manipulation of only the $x$ and $z$ coordinates. For example, the Data Mountain by Robertson et al. [20] was a $2 \frac{1}{2} \mathrm{D}$ environment; the items could be moved horizontally and in depth, but the $y$ location was locked, meaning that the items were "pushed" up along the slope of the mountain, and they always rested on this surface. The items could not be placed, for example, to hover a few inches above the surface of the mountain.

The method often used to evaluate the usability of 3D user interfaces is the memorize and retrieve task [10-12], which was designed largely to test the effectiveness of spatial memory in 3D environments. In the Task Gallery experiment, Robertson et al. [20] asked participants to create, modify and arrange tasks in space, a setup that more efficiently covers the idea of traditional user-experience testing. The variables measured most often are task completion times, incorrect attempts made before the correct retrieval, failed attempts and the number of correct retrievals. In this experiment, we used a modified version of the traditional memorize and retrieve task. Instead of spatial memory competence, we were more interested in how efficiently people search in stereoscopic 3D environments. To assess this parameter, we presented participants with a stereoscopic 3D image gallery application and asked them to locate a particular image by searching through the gallery. After every search, the order of images was randomized so that the participants were not able to utilize memorial cues.

\subsection{Objectives}

The study aimed to explore the effect of stereoscopic depth magnitude on the performance and user experience of a threedimensional image gallery using a 3-inch touch-screen autostereoscopic display. Our hypothesis was that having images in various depth planes would decrease visual crowding and make target detection easier.

\section{Methods}

\subsection{Displays}

Participants browsed images with an application run on a Texas Instruments OMAP Zoom Mobile Development Kit (Fig. 2). A 

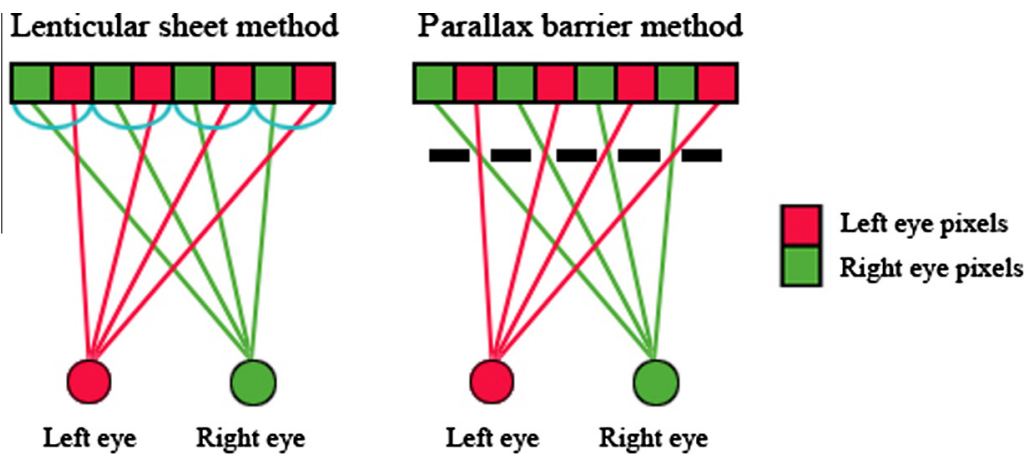

Fig. 1. (Left) Working principles of the lenticular sheet method. (Right) Principles of the parallax-barrier method.

lenticular lens sheet was attached over a TFT-LCD display (Table 1) to enable autostereoscopic viewing. The viewing distance was $35 \mathrm{~cm}$, and the valid viewing zone was $\pm 9.3^{\circ}$.

An additional display was utilized to show the participants the target images. The display was a $15.6^{\prime \prime}$ FHD LED PC screen that had a resolution of 1920 pixels horizontally and 1080 pixels vertically. The additional display was placed next to the OMAP device at a distance of $70 \mathrm{~cm}$ from the participant.

\subsection{Participants}

A total of 36 participants signed up for the experiment ( 5 males, mean age $=24.5, \mathrm{SD}=4.4$ ). They had no previous experience on the OMAP with stereoscopic touch screen. All participants had normal or corrected-to-normal vision and had no reported neurological disorders.

\subsection{Visual screening}

The participants completed a set of visual screening measurements prior to the experiments. This screening was performed to ensure that the participants had no major visual dysfunctions. The screening measurements included tests to assess the near and distance visual acuities, interpupillary distance, stereoscopic acuity, near point of convergence, near point of accommodation, lateral and vertical phorias and AC/C-ratio (accommodative convergence to convergence ratio).

Near and distance visual acuity were tested to ensure that the participant had sufficient visual capability to perform the test. The near vision acuity was measured with the LeaNumbers test No. 270900 [21], with a viewing distance of $40 \mathrm{~cm}$, and the distance visual acuity was tested with LeaNumbers test No. 271200 $[22,23]$, with viewing distance of $3 \mathrm{~m}$. Both tests utilized printed numbers for assessment of visual acuity. The test was performed

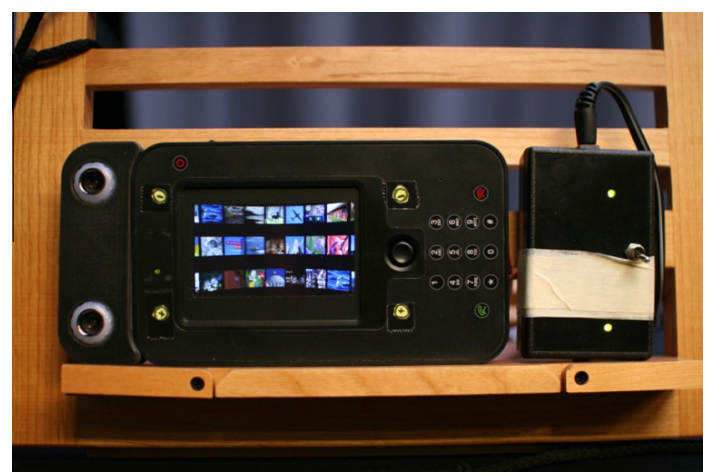

Fig. 2. OMAP Zoom Mobile Development Kit showing the image gallery.
Table 1

TFT-LCD specifications.

\begin{tabular}{ll} 
TFT-LCD & DX08D17VMOAAA, Hitachi, 3.07 WVGA \\
Module dimensions & $42.9 \mathrm{~mm}(\mathrm{~W}) \times 77.0 \mathrm{~mm}(\mathrm{H}) \times 1.59 \mathrm{~mm}(\mathrm{t})$ \\
Active area dimensions & $38.16 \mathrm{~mm}(\mathrm{~W}) \times 67.893 \mathrm{~mm}(\mathrm{H})$ \\
Pixel pitch & $0.0795 \mathrm{~mm}(\mathrm{~W}) \times 0.0795(\mathrm{H})$ \\
Resolution & $480 \times 3(\mathrm{R}, \mathrm{G}, \mathrm{B})(\mathrm{W}) \times 854(\mathrm{H})$ dots \\
Color pixel arrangement & RGB vertical stripe \\
Display mode & Transmissive type, normally black mode \\
\hline
\end{tabular}

for both eyes separately. In both tests, the participant was excluded from the experiment if the visual acuity was worse than 0.50 on either eye.

The interpupillary distance was measured by Nidek PM-600 pupillometer. A participant was accepted to the experiment if the interpupillary distance was between 51 and $71 \mathrm{~mm}$, corresponding to $90 \%$ of the normal population. The stereo acuity was measured with the TNO test [24], a stereo vision test that consists of anaglyph random-dot stereograms viewed at $40 \mathrm{~cm}$. The participant was accepted if his or her stereoscopic acuity was better than $240 \mathrm{~s}$ of arc. The lateral and vertical phorias were measured with the Maddox Wing test. The exclusion criteria in horizontal phoria were over 13 diopters in the exophoric direction or over 7 diopters in the esophoric direction [25]. In vertical phoria, the exclusion criterion was a vertical phoria of more than 1 diopter [25].

The AC/C-ratio was measured with the Maddox Wing test with lenses of +2.75 diopters added to the viewing holes. The near points of convergence and accommodation were tested with the RAF Gauge test. These points were tested to ensure that the muscular systems responsible for the convergence eye movements and accommodative changes in the lens of the eye were operating properly. The test results of these screening tests did not affect the experiment participation.

\subsection{Procedure}

Participants were seated in front of the OMAP device at a distance of $35 \mathrm{~cm}$. The additional display was immediately adjacent and had a viewing distance of $70 \mathrm{~cm}$. Distance and viewing angle were controlled with a chinrest. Fig. 3 demonstrates the experimental setup from the participant's point of view.

The user interface consisted of a S3D image gallery (Fig. 4) developed by the Astonishing Tribe $A B$ (Sweden). The image gallery was a convex curved wall to which were attached three rows of images, showing 21 images altogether. Each row had a different depth, the bottom row being the nearest and the top row the farthest, producing a layout resembling an amphitheater. The gallery could be rotated horizontally by using a stylus pen to drag the view on the touch screen. A larger view of a single image was shown 


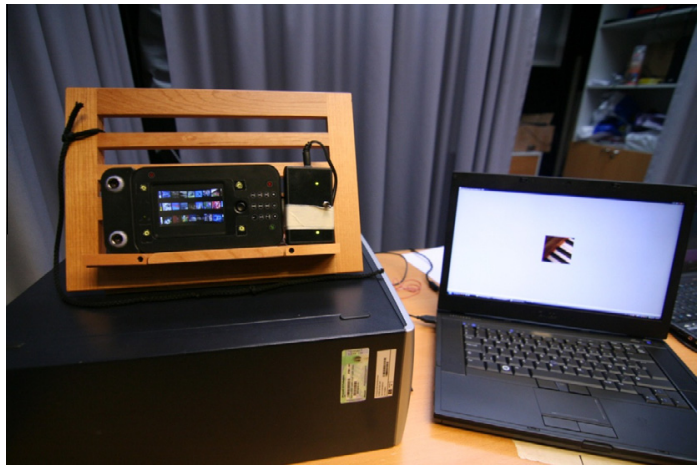

Fig. 3. Experimental setup with OMAP on the left and the additional display on the right.

when its thumbnail was clicked in the browser. Clicking the image again caused it to shrink back to a thumbnail.

Three depth magnitudes were used in the experiment: no depth, small depth and medium depth. In the small- and medium-depth conditions, the depth was uncrossed, i.e., farther from the viewer than the physical display surface. Each depth condition was tested in a separate block. The order of blocks was randomized. In the no-depth condition, there was no stereoscopic depth present in the image. However, monocular depth cues provided information about the curvedness of the rows. In the small-depth condition, the largest depth value in the middle of the display was $2.3 \mathrm{~min}$ of arc for the bottom row, $3.1 \mathrm{~min}$ of arc for the middle row and $3.9 \mathrm{~min}$ of arc for the top row (Table 2). In the mediumdepth condition, the largest depth value in the middle of the display was $4.7 \mathrm{~min}$ of arc for the bottom row, $6.2 \mathrm{~min}$ of arc for the middle row and $7 \mathrm{~min}$ of arc for the top row. The rows were curved in depth such that the depth gradually decreased from the maximum in the center to zero at the left and right edge of the display. Thus, there was always a range of depths from zero disparity to the maximum depth value at the middle of each row. The image size was the same in all conditions: horizontally and vertically $1.31^{\circ}$
Table 2

Depth values in the experiment.

\begin{tabular}{lll}
\hline & Small depth & Medium depth \\
\hline Min of arc & & \\
Top & 2.3 & 4.7 \\
Middle & 3.1 & 6.2 \\
Bottom & 3.9 & 7 \\
\hline
\end{tabular}

at the bottom row, $1.18^{\circ}$ at the middle row and $1.14^{\circ}$ at the top row.

Sixty images were utilized in the experiment. Half of them were from the USC-SIPI Image Database and from the Kodak Lossless True Color Image Suite (http://rOk.us/graphics/kodak/), and the rest of the images belonged to Per Grimberg or Oscar Olivestedt from The Astonishing Tribe AB. The image content was either scenery views or views of an object (a person, animal, plant or a tool/ vehicle). Different color and brightness levels were selected for the images. The goal was to have a wide range of color and brightness levels that would be comparable to a wide range of home photographs. Furthermore, we wanted to avoid any bias that might occur because a specific color or brightness level interacted with the autostereoscopic display. All participants saw the same set of images.

To determine how efficiently participants found images in the image gallery, we utilized a search task in the experiment. The participant was shown a target image $\left(256 \times 256\right.$ pixels; $3.76^{\circ}$ of visual angle) in the additional laptop display located adjacent to the stereoscopic display. The task of the participant was to search the target image in the image gallery. The participants browsed the OMAP display with a stylus, tapped the image open, and verbally indicated that they had found the correct image. If the participant picked an incorrect image, she or he continued the task until the correct image was found. Thus, error commission made the search time longer. At that point, the experiment leader pressed a key on the PC keyboard, which recorded the search time. The participant closed the image by tapping it again and pressed the asterisk $\left(^{*}\right)$ button on the OMAP device to randomize the order of the images.

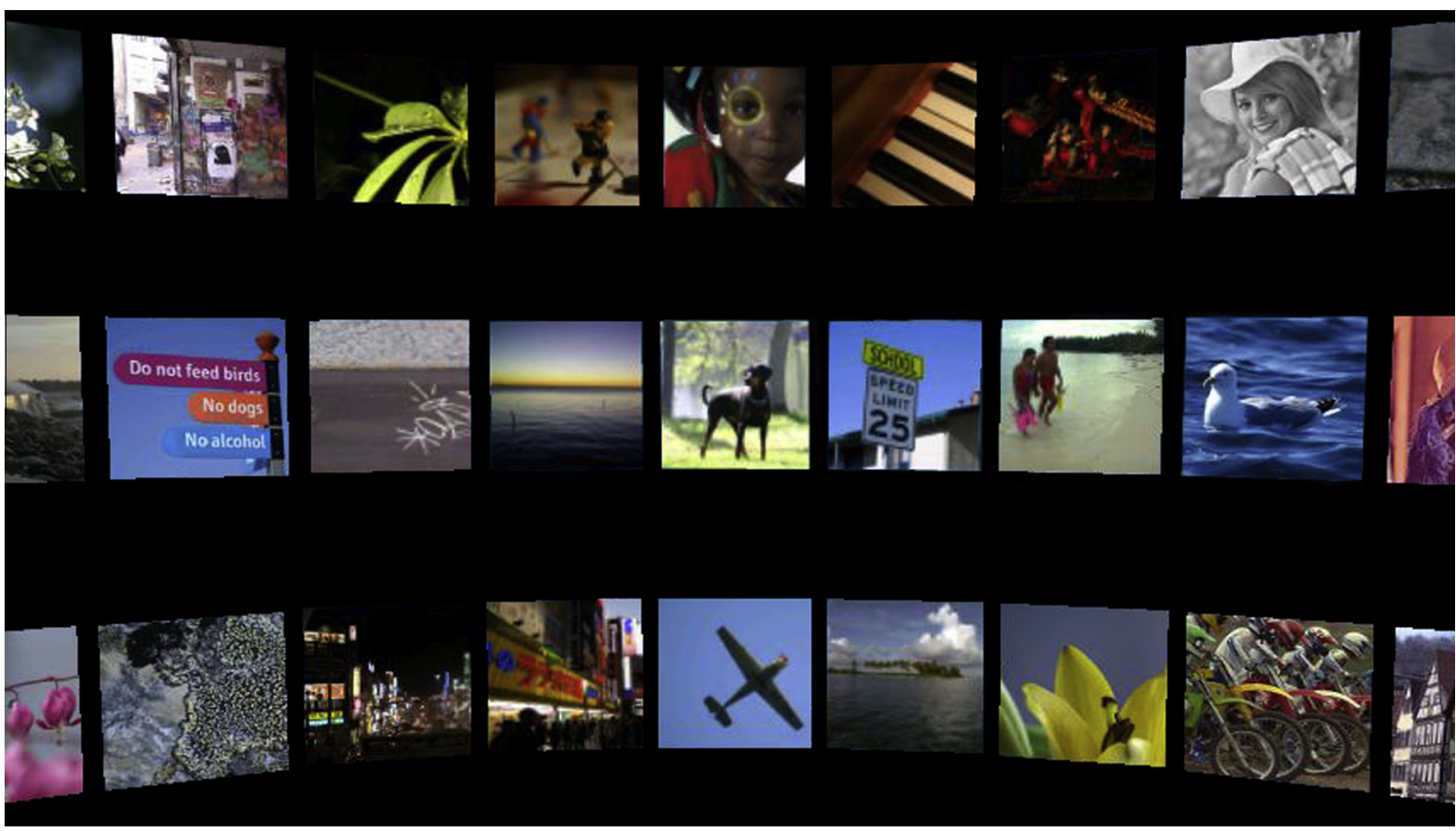

Fig. 4. The gallery layout. 
The laptop then displayed a new target image. The search was performed a total of 30 times, 10 times for each layout: no depth, small depth and medium depth. The presentation order of the layouts was randomized for each participant.

Each series of 10 consecutive searches consisted of a single layout, after which participants filled in a questionnaire focusing on the perceived visual quality of the layout and on the usability of the software. The questionnaire included six Likert scales ranging from 1 to 10: (1) overall image quality, (2) satisfaction when using the software, (3) layout clarity (for example, how well the pictures were perceived), (4) "coolness" of the 3D effect, (5) usefulness of the 3D effect and (6) visual load of the 3D effect (eye strain/headache). In the last three scales, the term "3D" was removed in the no-depth condition. After the subjective scales, the next layout was tested with 10 searches, and the subjective scales were filled again. Ultimately, after the three series of searches, the participants were asked to compare the layouts and to give free comments on the application in general by writing.

\section{Results}

The PASW-19 statistics software (SPSS, Inc.) was used for analysis. Table 3 presents the mean and standard deviation in each of the cases. Fig. 5 presents the mean search time and 95\% confidence interval for each layout condition. The data distributions were positively skewed in each of the cases, so the search times were corrected by natural logarithm for the analysis of variance (ANOVA). One participant had search times that were more than three standard deviations slower compared with other participants, so he was excluded from further analysis. A repeated measures ANOVA revealed a significant main effect for layout $[F(2,68)=3.86, p<.05$, Partial eta-squared $=0.10]$. Pairwise comparisons with Bonferroni-corrected $t$-tests revealed that there was a significant difference between the no-stereo and smalldepth conditions $(p<0.05)$.

\subsection{Subjective measurements}

Fig. 6 presents the mean ratings for the six questions that were asked after each layout condition. A repeated measures ANOVA indicated that the effect of layout on the ratings was significant with respect to image quality $(p<0.05)$, "coolness" $(p<0.001)$, usefulness $(p<0.001)$ and eye strain $(p<0.001)$. Pairwise comparisons with Bonferroni-corrected $t$-tests indicated differences in several questions. The condition without stereoscopic depth had significantly lower ratings on coolness, usefulness of visualization and eye strain. Furthermore, the eye-strain difference between small- and medium-depth conditions approached significance $(p<0.10)$.

\subsection{Open evaluations of the layouts}

Open evaluations were analyzed and coded thematically. In Table 4, the most common themes are cross-tabulated with the experimental conditions. Plus and minus signs are used to indicate whether the comment is positive or negative. The first columns on the left indicate general comments, and the subsequent columns

Table 3

Mean and standard deviation values.

\begin{tabular}{lll}
\hline Experimental condition & Mean & Standard deviation \\
\hline No depth & 8.88 & 3.11 \\
Small depth & 7.22 & 2.24 \\
Medium depth & 8.41 & 3.55 \\
\hline
\end{tabular}

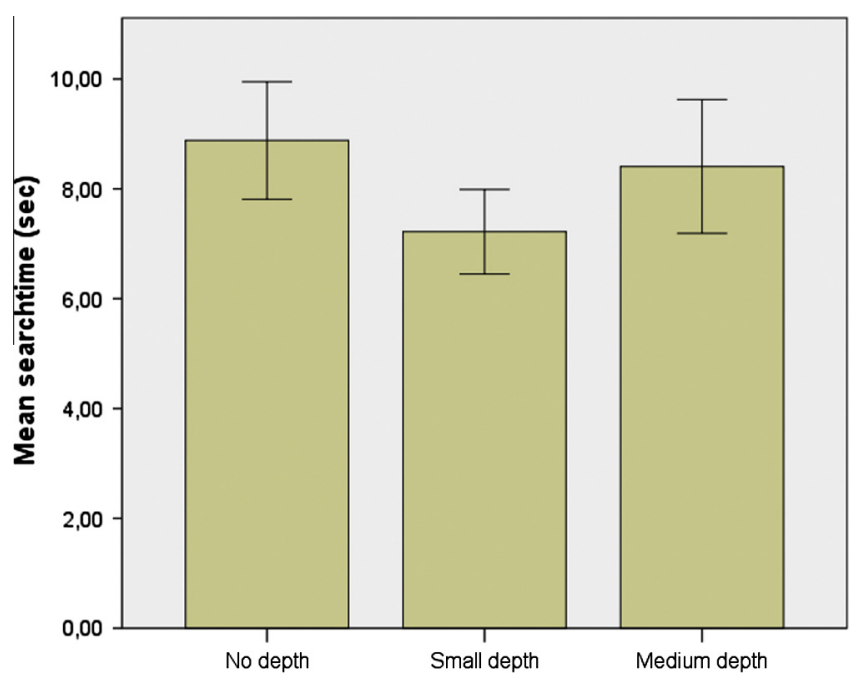

Fig. 5. Mean search times for no-depth, small-depth, medium-depth and amphitheater layouts. Error bars depict 95\% confidence intervals.

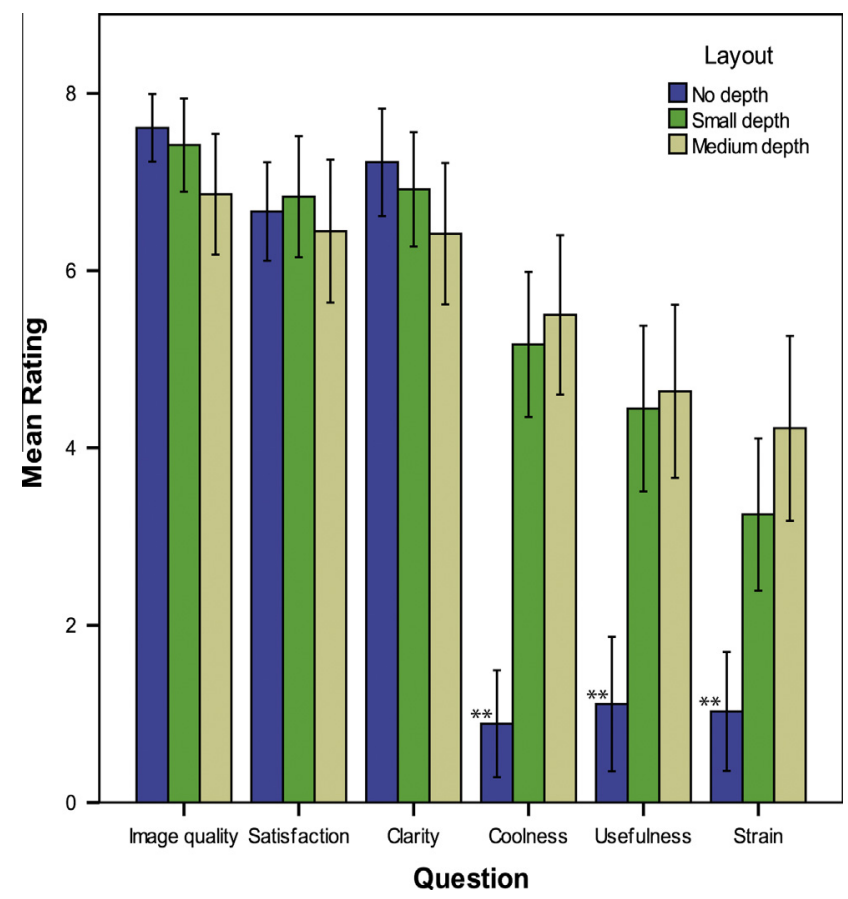

Fig. 6. Mean evaluation values of the three different layouts. An asterisk above a single bar indicates that the indicated condition is significantly different from all other conditions. Error bars depict 95\% confidence intervals.

exhibit comments related to small-, medium- and no-depth conditions. The most commonly mentioned themes were layout clarity (total frequency 14), usefulness of stereoscopic visualization $(f=11)$, ease of use $(f=9)$ and distinctiveness of the images $(f=9)$. Stereoscopic layouts received more positive feedback (17 positive/5 negative comments)than the $2 \mathrm{D}$ layout did (4 positive/ 8 negative comments). Eye strain was mentioned only four times with stereoscopic versions of the layout.

Typical comments were as follows:

- "The small depth and the small-depth layout were good and clear. " (Layout clarity).

- "The small depth looked the best and was easiest and fastest to use." (Ease of use). 
Table 4

Frequencies of different themes.

\begin{tabular}{|c|c|c|c|c|c|c|c|c|c|c|c|}
\hline \multirow[t]{2}{*}{ Themes } & \multicolumn{2}{|c|}{ General } & \multicolumn{2}{|c|}{ Medium } & \multicolumn{2}{|c|}{ Small } & \multicolumn{2}{|c|}{ No depth } & \multicolumn{2}{|c|}{ Sum } & \multirow[t]{2}{*}{ Total } \\
\hline & + & - & + & - & + & - & + & - & + & - & \\
\hline Layout clarity & 4 & 2 & 4 & & 3 & & & 1 & 11 & 3 & 14 \\
\hline Usefulness of S3D & 4 & 7 & & & & & & & 4 & 7 & 11 \\
\hline Ease of use & 1 & & 2 & 1 & 2 & & 2 & 1 & 7 & 2 & 9 \\
\hline Distinctiveness & 4 & 2 & & & 2 & & & 1 & 6 & 3 & 9 \\
\hline Pleasantness & & & 1 & 1 & 1 & & 1 & 2 & 3 & 3 & 6 \\
\hline Coolness of S3D & 2 & 2 & & & & & & 1 & 2 & 3 & 5 \\
\hline Smooth browsing & 1 & 2 & 1 & & & & 1 & & 3 & 2 & 5 \\
\hline Eye strain & & 1 & & 1 & 1 & 1 & & & 1 & 3 & 4 \\
\hline Image jitter & & 1 & & & & 1 & & 1 & & 3 & 3 \\
\hline Cannot see 3D effect & & & & & & & & 1 & & 1 & 1 \\
\hline Sum & 16 & 17 & 8 & 3 & 9 & 2 & 4 & 8 & 37 & 30 & 67 \\
\hline
\end{tabular}

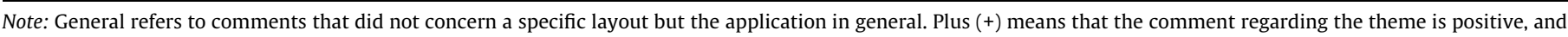
minus (-) means that the comment is negative.

- "2D pictures were harder to find, did not differentiate from one another so well." (Distinctiveness of pictures).

- "Stereoscopic effect was not exactly useful or helpful, but it made the use of the software more interesting" (Usefulness of 3D).

\section{Discussion}

Stereoscopic depth produces performance benefits with small autostereoscopic touch displays; the results revealed that the images were found faster in the layout with small depth than in the no-depth and medium-depth conditions. The effect may be related to visual crowding, which has been shown to decrease between images at different depths [4]. Reduced crowding might lead to more efficient visual search strategies, as images are easier to detect. However, the beneficial effect disappears quickly if the depth is increased, which might be related to display features, perception or interaction. It is possible that the crosstalk increase as a function of disparity, which would make the identification of the images more difficult. Questionnaire results (Fig. 6) indicate that perceived image quality decreased with increasing disparity. However, the difference is not statistically significant, so it is difficult to define the importance of this factor in slowing the search times. This effect should be carefully controlled in future experiments by using a stereoscopic system that has less crosstalk than the autostereoscopic display that we used in the current experiment.

The results might also be related to the visual processing of stereoscopic information. It takes the user a longer time to switch between the depth planes when the depth magnitude is higher. However, the depth differences in our experiment were small, so it is not probable that this caused the result. Furthermore, the relationship of stereoscopic display elements might have affected the result. In the current setup, there were used uncrossed depth values, as the far depth produces less frame violation problems with small mobile displays. However, this setup might have affected the search speed, as it has been shown that the search efficiency varies when three-dimensional configuration is changed [26]. For example, it is faster to search for a close target among far targets than a far target among close targets. These results were acquired with random-dot stereograms, so it is not clear how well they can be applied to other stereoscopic images. This is clearly an issue for further research.

Additionally, all the disparity values in both stereoscopic conditions were small. This condition partly explains why the search times in our experiment were long in comparison to earlier results demonstrating efficient searching for stereoscopic targets $[7,27]$. However, de La Rosa et al. [8] reported that rapid search was possible only when the stimuli had a disparity of eight arc minutes or more. It would be interesting to test in future studies whether significantly higher stereoscopic depth values would affect the search speed in the stereoscopic touch display settings. At the same time, it would be important to have the depth of a single row at the same depth level so that the depth factor would be better controlled.

Stereoscopic depth might also affect touch interaction, which becomes more difficult with increasing depth because the discrepancy between the location of the physical display surface and the stereoscopically presented image increases. As the participants must tap the stylus on the physical display surface in front of the virtual image, the possibility of tapping errors and frustration may increase. This interaction is also an effect that should be further studied in experiments that control the depth magnitude and visual properties of user-interface elements.

The questionnaire results revealed that participants regarded the stereoscopic 3D user-interface as "cooler" and more useful than the non-stereoscopic version of the same interface. Conversely, the stereoscopic presentation produced more eye strain compared with the non-stereoscopic layout. However, the rated eye-strain values were moderate only in the small-depth (mean rating of 3.2 out of 10) and medium-depth conditions (mean rating of 4.2) as compared with the rated eye strain in non-stereoscopic version (mean rating of 1.0 ).

In conclusion, our study demonstrated that using small S3D may enhance the performance in visual search task on an autostereoscopic mobile display if a moderate depth magnitude is used. Thus, the inclusion of a small 3D effect could be useful for browsing pictures and possibly for other types of navigation as well, where a large amount of information is presented to the user on a small display. Further investigations are needed to fully understand the mechanisms facilitating performance with a small 3D effect.

\section{Acknowledgements}

We thank Dan Gärdenfors (the Amazing Tribe AB, currently at Research in Motion) for his help in the process of designing the user interface. The project was funded by the European Union FP7 3DPhone project (FP7-213349) and the Academy of Finland Computational Psychology of Experience in HCI project (COPEX; project number 264323).

\section{References}

[1] R. Rajae-Joordens, Measuring Experiences in Gaming and tv Applications, in: J. Westerink, M. Ouwerkerk, T. Overbeek, W. Pasveer, B. de Ruyter, B. (Eds.), Probing experience, Philips Research Book Series, Springer, Netherlands, 2008, pp. 77-90.

[2] S.L. Wu, Depth in dedicated mobile device user interfaces for auto-stereoscopic displays. Master's Thesis, Delft University of Technology, Netherlands, 2010 
[3] P.J. Seuntiëns, I. Heynderickx, W. IJsselsteijn, P.M.J. van den Avoort, J. Berentsen, I. Dalm, M. Lambooij, W. Oosting, Viewing experience and naturalness of 3D images, Proc. SPIE 6016 (2005) 43-49.

[4] F.M. Felisberti, J.A. Solomon, M.J. Morgan, The role of target salience in crowding, Perception 34 (2005) 823-833.

[5] K. Nakayama, S. Shimojo, G.H. Silverman, Stereoscopic depth: its relation to image segmentation, grouping, and recognition of occluded objects, Perception 18 (1989) 55-68.

[6] S. Mizobuchi, S. Terasaki, J. Häkkinen, E. Heinonen, J. Bergquist, M. Chignell, The effect of stereoscopic viewing in a word-search task with a layered background, J. SID (16) (2008) 1105-1113.

[7] Z.J. He, K. Nakayama, Visual attention to surfaces in three-dimensional space, PNAS 92 (1995) 11155-11159.

[8] S. de la Rosa, G. Moraglia, B. Schneider, The magnitude of binocular disparity modulates search time for targets defined by a conjunction of depth and colour, Can. J. Exp. Psych. 62 (2008) 150-155.

[9] F.L. Kooi, A. Toet, S. Tripathy, D.M. Levi, The effect of similarity and duration on spatial interactions in peripheral vision, Spat. Vis. 8 (1994) 255-279.

[10] M. Tavanti, M. Lind, 2D vs 3D, Implications on Spatial Memory, in: Proc. IEEE Symp.Inf. Vis., 2001, pp. 139-145.

[11] A. Cockburn, B. McKenzie, Evaluating the Effectiveness of Spatial Memory in 2D and 3D Physical and Virtual Environments, in: Proc.CHI, 2002, pp. 20-25.

[12] A. Cockburn, Revisiting 2D vs 3D Implications on Spatial Memory, in: Proceedings of the fifth Conference on Australasian user Interface, 2004, pp. 25-31.

[13] Y.Y. Yeh, L.D. Silverstein, Limits of fusion and depth judgment in stereoscopic color displays, Hum. Factors 32 (1990) 45-60.

[14] M. Lambooij, W. IJsselsteijn, M. Fortuin, I. Heynderickx, Visual Discomfort and visual fatigue of stereoscopic displays: a review, J. Imaging Sci. Technol. 53 (2009) 1-14.

[15] J. Häkkinen, J. Takatalo, M. Kilpeläinen, M. Salmimaa, G. Nyman, Determining limits to avoid double vision in an autostereoscopic display: disparity and image element width, J. SID 17 (2009) 433-441.
[16] J. Häkkinen, T. Kawai, J. Takatalo, T. Leisti, J. Radun, A. Hirsaho, G. Nyman, Measuring Stereoscopic Image Quality Experience with Interpretation based Quality Methodology, in: Proc. SPIE 6808, 2008, pp. 68081B-68081B-12.

[17] D. Valkov, F. Steinicke, G. Bruder, 2D Touching of 3D Stereoscopic Objects, in: Proc.CHI, 2011, pp. 1353-1362.

[18] Frank L. Kooi, Alexander Toet, Visual comfort of binocular and 3D displays, Displays 25 (2004) 99-109.

[19] I. Tsirlin, L.M. Wilcox, R.S. Allison, The effect of crosstalk on the perceived depth from disparity and monocular occlusions, IEEE Trans. Broadcast. 57 (2011) 445-453.

[20] G. Robertson, M. Czerwinski, K. Larson, D. Robbins, D. Thiel, M. van Dantzich Data Mountain: Using Spatial Memory for Document Management, in: Proc. 11th annual ACM Symp. User Interface software and technology, 1998, pp. 153-162.

[21] Internet page <http://www.lea-test.fi/index.html?start=en/vistests/instruct/ 2709-10/index.html> Accessed 2.4.2014

[22] Internet page <http://www.lea-test.fi/index.html?start=en/vistests/instruct/ 2701/index.html> Accessed 2.4.2014.

[23] J.J. Vaidhyan, P. Lietzen, D. Ah-Kine Ng Poon Hing, A. Pathak, E. Ahonen, N. Quinn, S. Lyons, M. Leinonen, L. Hyvarinen, B. Moore, Comparison of visual acuity measured with lea symbols and lea numbers to adult standards, Invest. Ophthalmol. Visual Sci. 48 (2007) (E-Abstract 4853).

[24] J. Walraven, Amblyopia screening with random-dot stereograms, Am. J. Ophthalmol. 80 (1975) 893-900.

[25] E. Peli, The visual effects of head-mounted display (HMD) are not distinguishable from those of desk-top computer display, Vision Res. 38 (1998) 2053-2066.

[26] A.J. O'Toole, C.L. Walker, On the preattentive accessibility of stereoscopic disparity: evidence from visual search, Percept. Psychophys. 59 (1997) 202 218.

[27] K. Nakayama, G.H. Silverman, Serial and parallel processing of visual feature conjunctions, Nature 320 (1986) 264-265. 